\title{
Calculation of pre-equilibrium effects in neutron-induced cross section on ${ }^{32,34} \mathrm{~S}$ isotopes using the EMPIRE 3.2 code
}

\author{
Leila Yettou ${ }^{a}$, Mohamed Belgaid \\ University of Bab Ezzouar, Faculty of Physics, Laboratory SNIRM, Algiers, Algeria
}

\begin{abstract}
In this study, a new version EMPIRE 3.2 code was used in the cross section calculations of (n,p) reactions and in the calculation of proton emission spectra produced by $(n, x p)$ reactions. Exciton model predictions combined with the Kalbach angular distribution systematics were used and some parameters such as those of mean free path, cluster emission in terms of Iwamoto-Harada model, optical model potentials of Morillon for neutrons and protons in the energy range up to $20 \mathrm{MeV}$, level density for spherical nuclei of Gilbert-Cameron model and width fluctuation correction in terms of compound nucleus have been investigated our calculations. The excitation functions and the proton emission spectra for ${ }^{32,34} \mathrm{~S}$ nuclei were calculated, discussed and found in good agreement with available experimental data.
\end{abstract}

\section{Introduction}

Neutron induced reactions on ${ }^{32,34} \mathrm{~S}$ isotopes and double differential cross sections calculations for proton emission are important not only for many materials as requested by the accelerator driven systems (ADS) and waste transmutation problems but also for applications of radioactivity in both diagnostics and therapy $[1,2]$. According to Gupta et al., [3], pre-equilibrium processes play an important role in nuclear reactions induced of few $\mathrm{MeV}(<50 \mathrm{MeV})$ where their influences at $14.8 \mathrm{MeV}$ have been studied. Experimental neutrons induced cross section data accessed by the EXFOR database [4] are necessary to develop the theoretical models. These models are frequently needed when the experimental data are not obtained because of the experimental difficulties. The main purpose of this work is to investigate the sensitivity to input parameters in neutron-induced reactions in the energy range up to $20 \mathrm{MeV}$ by using the EMPIRE 3.2 code [5].

\section{Exciton model formulae}

The module PCROSS included in the EMPIRE 3.2 code [5] describes the classical exciton model [6] which includes nucleon, cluster and gamma emissions. This model is based on the solution of the master equation [7] in the form proposed by Cline $[8,9]$ as:

$$
\begin{aligned}
-q_{t=0}(n) & =\lambda_{+}(E, n+2) \tau(n+2) \\
& +\lambda_{-}(E, n-2) \tau(n-2) \\
& -\left[\lambda_{+}(E, n)+\lambda_{-}(E, n)+L(E, n)\right] \tau(n)
\end{aligned}
$$

where $q_{t}(n)$ is the initial occupation probability of the composite nucleus in the state with the exciton number $n$, $\lambda_{+}(E, n)$ and $\lambda_{-}(E, n)$ are the transition rates for decay to neighboring states, and $L(E, n)$ is the total emission rate integrated over emission energy for particles (protons $\pi$, neutrons $v$ and clusters) and $\gamma$-rays. The pre-equilibrium spectra can be calculated as:

$$
\begin{aligned}
\frac{d \sigma_{a, b}}{d \epsilon_{b}}\left(\epsilon_{b}\right) & =\sigma_{a, b}^{r}\left(E_{i n c}\right) D_{a, b}\left(E_{\text {inc }}\right) \\
& \times \sum_{n} W_{b}\left(E, n, \epsilon_{b}\right) \tau(n)
\end{aligned}
$$

where $\sigma_{a, b}^{r}\left(E_{\text {inc }}\right)$ is the cross section of the reaction $(a, b)$ , $W_{b}\left(E, n, \epsilon_{b}\right)$ is the probability of emission of a particle of type $b$ (or gamma ray) with energy $\epsilon_{b}$ from a state with $n$ excitons and excitation energy $E$ of the $\mathrm{CN}$, and $D_{a, b}\left(E_{i n c}\right)$ is the depletion factor. The PCROSS code uses the Williams formula [10], where the Pauli correction $A(p, h)$ is calculated in accordance with Kalbach's method [11].

$$
\omega(p, h, E)=g \frac{(g(E-D)-A(p, h))^{p+h-1}}{p ! h !(p+h-1) !}
$$

Using the parameterization of transition rate proposed by Blann and Mignerey [12] and the particle-hole state densities from the Williams formula, we obtain the expressions for the internal transition rates found by Machner [13].

\footnotetext{
${ }^{a}$ Corresponding author: yettouleila@yahoo.fr
} 


$$
\lambda_{+}(E, n)=\frac{1}{K_{m f p}}\left[1.4 \times 10^{21} E^{\prime}-\frac{2}{n+1} 6 \times 10^{18} E^{\prime 2}\right]
$$

where $K_{m f p}$ is the mean free path parameter which set to a value of 1.5 (by default) in our calculations. Kalbach's method $[7,8,13]$ was implemented for the calculation of the nucleon emission rate. The probability of emission $W_{b}\left(E, n, \epsilon_{b}\right)$ of a nucleon $b$ with spin $s_{b}$, reduced mass $\mu_{b}$ and energy $\epsilon_{b}$ from a state with $n$ excitons is given by,

$$
\begin{aligned}
W_{b}\left(E, n, \epsilon_{b}\right)= & \frac{2 s_{b}+1}{\pi^{2} \hbar^{3}} \mu_{b} \epsilon_{b} \sigma_{b}^{i n v}\left(\epsilon_{b}\right) \\
& \times \frac{\omega_{r e s}(p-b, h, U)}{\omega_{C N}(p, h, E)} Q_{b}(p, h)
\end{aligned}
$$

where $E(U)$ is the excitation energy of the $\mathrm{CN}$ (residual nucleus), $\omega(p, h, U)$ is the particle-hole state density, and $\sigma_{b}^{i n v}$ is the inverse channel reaction cross section. The factor $Q_{b}(p, h)$ takes into account the fraction of $b$ nucleons in the $n$-th stage of the reaction and is calculated as discussed by Gupta [14]. In the framework of statistical model (compound nucleus model), the Hauser-Feshbach model was used.

The decay probability (Eq.6) is defined in terms of transmission coefficients associated to the reaction channels which might be particles emission, photon emission or fission.

$$
P_{b}(E, J \pi)=\frac{T_{a}\left(E_{x}, J \pi\right)}{\sum_{c} T_{c}\left(E_{x}, J \pi\right)}
$$

In the EMPIRE 3.2 code [5], the level densities are described by several models with the corresponding parameterizations. The phenomenological GilbertCameron Model [15], which is included in RIPL-3 library [16] is used in this work and the spin cut-off factor $\sigma\left(E_{x}\right)$ is given by:

$$
\sigma^{2}\left(E_{x}\right)=0.146 A^{2 / 3} \sqrt{a U}
$$

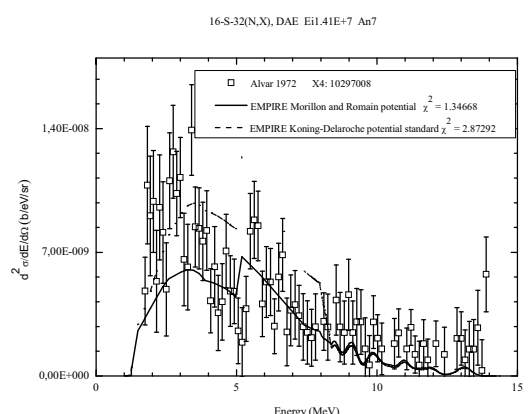

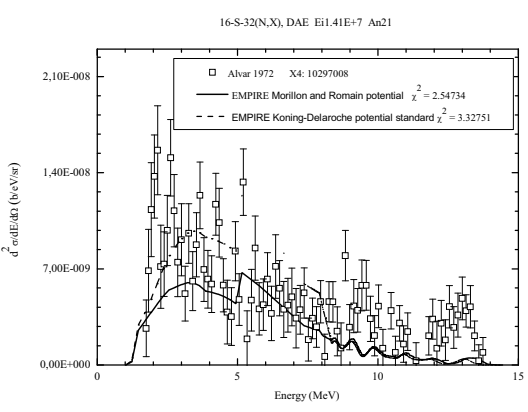
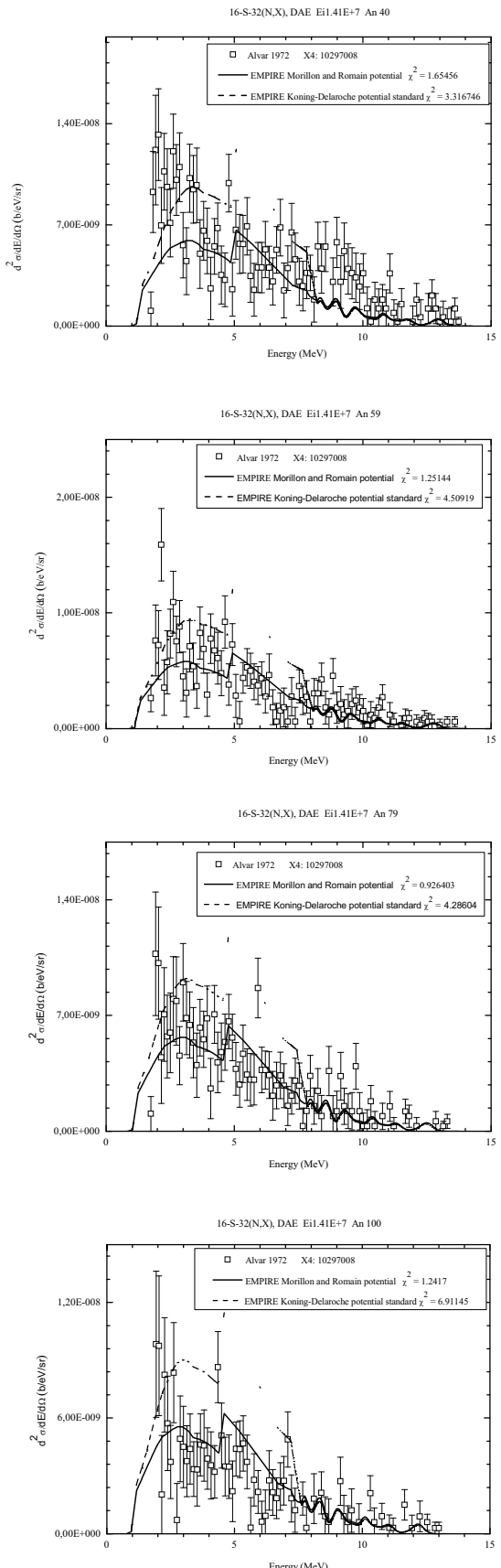


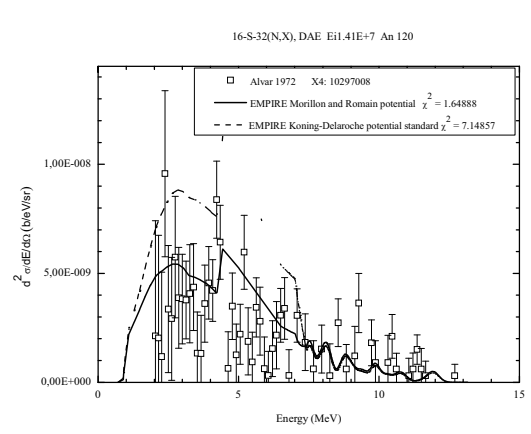

Fig. 1 Calculated double differential cross sections at various angles for ${ }^{32} \mathrm{~S}(\mathrm{n}, \mathrm{x}){ }^{32} \mathrm{P}$ reactions at $14.1 \mathrm{MeV}$ incident neutron energy using Morillon and Romain potential [19,20] (continuous lines) compared to the Koning-Delaroche potential standard [21] (dashed lines) and to the experimental data (open squares) [1].

\section{Results and discussions}

In this work, the theoretical calculations have been made in the framework of exciton model [6] combined with Kalbach angular distributions systematics [17]. Probability of cluster emission is calculated in terms of the IwamotoHarada model [18]. The mean free path parameter of the nucleon in the nuclear matter [12] set to 1.5 (by default) in PCROSS module. Hauser-Feshbach calculations require transmission coefficients for particle emission, for energies spanning from zero to the maximum emission energy. The sulfur dispersive global spherical optical model potential of Morillon [19, 20] was used both for neutrons and protons. The dispersive potentials with different geometry of the imaginary and real parts are used with the ECIS module of the EMPIRE 3.2 code [5]. The Gilbert-Cameron model [15] nuclear level densities were used which include the level density $a$-parameter of Arthur systematics [22] in order to perform the calculations. Theoretical predictions based on the exciton model combined with the Kalbach angular distribution systematics [17] are shown as continuous lines for all the figures. The calculated double differential emission spectra at various angles for ${ }^{32} \mathrm{~S}(\mathrm{n}, \mathrm{x}){ }^{32} \mathrm{P}$ reactions at 14.1 $\mathrm{MeV}$ incident neutron energy using the Morillon and Romain potential [19, 20] (continuous lines) compared to the Koning-Delaroche potential standard [21] (dashed lines) and to the experimental data [1] (open squares) are shown in Fig. 1. The different free parameters used by default in PCROSS module of the EMPIRE 3.2 code [5] and the dispersive global spherical optical model potential of Morillon for both neutrons [19] and protons [20] and the Gilbert-Cameron model [15] nuclear level densities were sufficient to fit the proton emission at $14 \mathrm{MeV}$ incident energy in Figs. 1 and 2 respectively. Also, the Morillon and Romain potential for neutrons [19] and protons [20] give the lower value of $\chi^{2}$ when compared to the KoningDelaroche potential standard for neutrons and protons [21] and when compared to the experimental data [1] at all emission angles. The calculated excitation functions and the experimental data [24-33] for ${ }^{32} \mathrm{~S}(\mathrm{n}, \mathrm{p})^{32} \mathrm{P}$ and ${ }^{34} \mathrm{~S}(\mathrm{n}, \mathrm{p}){ }^{34} \mathrm{P}$ reactions are shown in Figs. 3 and 4 respectively as a function of neutron induced energy in the range of 12-20 MeV. The sensitivities of the level density $a$-parameter of Arthur systematics [22] of GilbertCameron [15] and the dispersive global spherical optical model potential of Morillon and Romain [20] for both neutrons and protons give a good agreement between the calculations and the experimental data.

\section{Conclusion}

In this study, the results of the calculated double differential cross sections for ${ }^{32} \mathrm{~S}(\mathrm{n}, \mathrm{x}){ }^{32} \mathrm{P}$ at $14 \mathrm{MeV}$ incident energy agree well with the experimental data [1, 23 ] by using the Morillon and Romain potential of the EMPIRE 3.2 code [5]. Also, the excitation curves for ${ }^{32} \mathrm{~S}(\mathrm{n}, \mathrm{p}){ }^{32} \mathrm{P}$ and ${ }^{34} \mathrm{~S}(\mathrm{n}, \mathrm{p}){ }^{34} \mathrm{P}$ reactions exhibit better agreement with the level density $a$-parameter according to Arthur systematics [22] of Gilbert-Cameron [15]. Finally, our calculations using both classical exciton model [6] and Hauser-Feshbach theory describe the experimental data well. The reasons can be that the light nucleus of sulfur and/or the energy range up to $20 \mathrm{MeV}$ are sufficient to fit the curves. We hope others data above $20 \mathrm{MeV}$ from the EXFOR database [4] in order to show the pre-equilibrium effect of exciton model [6] in neutron-induced cross section on ${ }^{32,34} \mathrm{~S}$ isotopes using the EMPIRE 3.2 code [5].

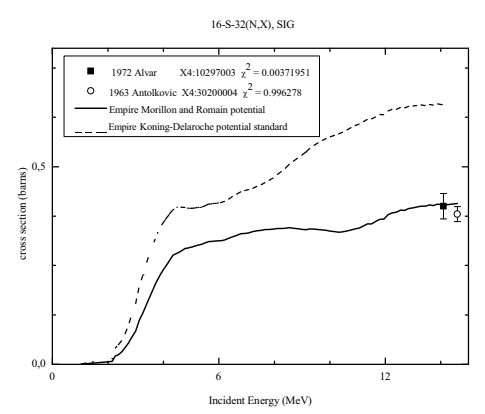

Fig.2 The comparison of the calculated proton emission cross section (continuous lines) with the Morillon and Romain potential [19, 20] (continuous lines), the Koning-Delaroche potential standard [21] (dashed lines) and the experimental data at $14.1 \mathrm{MeV}$ and $14.6 \mathrm{MeV}$ neutron incident energies for ${ }^{32} \mathrm{~S}(\mathrm{n}$, $\mathrm{x})^{32} \mathrm{P}$ reaction. The experimental data (open square and open lozenge) are taken from the references [1] and [23].

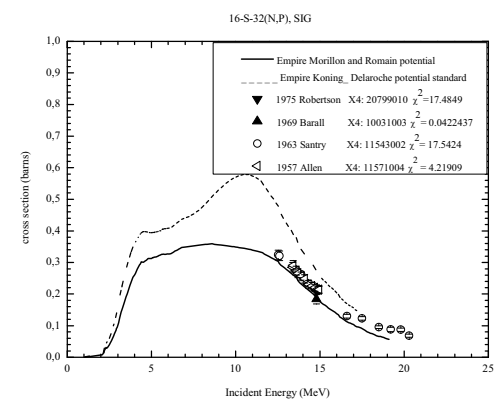

Fig. 3 The comparison of the excitation function for ${ }^{32} \mathrm{~S}(\mathrm{n}, \mathrm{p})^{32} \mathrm{P}$ reaction using Morillon and Romain potential [20] (continuous lines) compared to the Koning-Delaroche potential standard [21] (dashed lines) and to the experimental data [24-27]. 


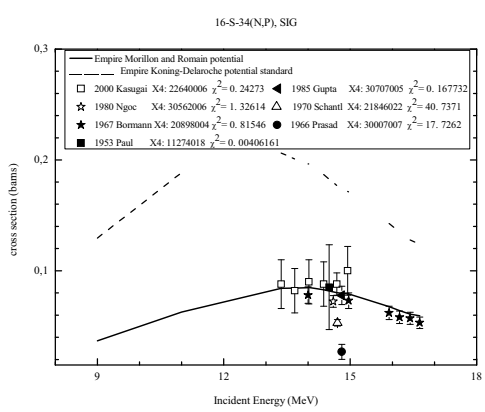

Fig.4 The comparison of the excitation function for ${ }^{34} \mathrm{~S}(\mathrm{n}, \mathrm{p}){ }^{34} \mathrm{P}$ reaction using Morillon and Romain potential [20] (continuous lines) compared to the Koning-Delaroche potential standard [21] (dashed lines) and to the experimental data [28], [3], [29-33].

\section{Acknowledgments}

We thank the organizers of the workshop (IAEA) 2-6 dec2013 for this opportunity to offer us view of the EMPIRE 3.2 code.

\section{References}

1. K.R. Alvar, Proton energy and angular distributions from $(n, p)$ and $(n, n p)$ reactions. Nuclear Physics A 195, 1, 289-301, (1972).

2. S.M. Qaim et al., Nuclear data for the Production of Therapeutic Radionuclides. International Atomic Energy Agency, Vienna, (2011).

3. J.P. Gupta, H.D. Bhardwaj, R. Prasad, Preequilibrium emission effect in $(n, p)$ reaction crosssections at $14.8 \mathrm{MeV}$. Pramana 24, 4, 637-642, (1985).

4. Experimental Nuclear Reaction Data (EXFOR), https:/www-nds.iaea.org/exfor/exfor.htm.

(Database Version of March 16, 2015).

5. Nuclear Reaction Model Code System for Data Evaluation. IAEA and NNDC Web sites post EMPIRE-3.2 (Malta), http://www.nndc.bnl.gov/ empire/, (2013).

6. J.J. Griffin, Phys. Rev. Lett. 17, 478, (1966).

7. C.K. Cline and M. Blann, Nucl. Phys. A 172, 225, (1971).

8. C.K. Cline, Nucl. Phys. A 193, 417, (1972).

9. I. Ribansky, P. Oblozinsky, and E. Betak, Nucl. Phys. A 205, 545, (1973).

10. F.C. Williams, Nucl. Phys. A 166, 23, (1971).

11. C. Kalbach, In Proc. of the IAEA Advisory Group Meeting on Basic and Applie Problems of Nuclear Level Densities, edited by E.M.R. Bhat (Brookhaven National Lab Report No. BNL-NSC-51694, Upton, NY), 113, (1983).

12. M. Blann and A. Mignerey, Nuclear Physics A 186, 245, (1972).

13. H. Machner, Z. Phys A 302, 125, (1981).
14. S. K. Gupta, Z. Phys. A 303, 329, (1981).

15. A. Gilbert and A.G.W. Cameron, Can. J. Phys. 43, 1446, (1965).

16. R. Capote, et al. Reference Input Parameter Library (RIPL-3). Nuclear Data Sheets 110, 12, 3107-3214, (2009).

17. C. Kalbach, Phys. Rev. C 37, 2350, (1988).

18. A. Iwamoto and K. Harada, Phys. Rev. C 26, 182, (1982).

19. B. Morillon, and P. Romain, Phys. Rev. C 70, p. 014601, (2004).

20. B. Morillon and P. Romain, Phys. Rev. C 76, p. 044601 (2007).

21. A.J. Koning, J.P. Delaroche, Nucl. Phys. A713, 231 (2003).

22. P.G. Young et al., Trans. Amer. Nucl. Soc. 60, 271, (1989).

23. B. Antolković, Protons from ${ }^{32} \mathrm{~S}$ bombarded by 14.4 MeV neutrons. Journ.: Nuovo Cimento 22, 4, 853 855, (1961).

24. J.C. Robertson, B. Audric and P. Kolkowski, Some neutron activation cross-sections at $14.78 \mathrm{MeV}$. Journal of Nuclear Energy 27, 8, 531-541, (1973).

25. R.C. Barrall, M. Silbergeld and D.G. Gardner, Cross sections of some reactions of $\mathrm{Al}, \mathrm{S}, \mathrm{Mn}, \mathrm{Fe}, \mathrm{Ni}$, In and I with $14.8 \mathrm{MeV}$ neutrons. Nuclear Physics A, 138, 2, 1, 387-391, (1969).

26. D.C. Santry, J.P. Butler, The $\mathrm{S}^{32}(n, p) \mathrm{P}^{32}$ reaction as a fast-neutron flux monitor, revue canadienne de chimie, 41(1): 123-133, (1963).

27. L.Jr. Allen, et al., Cross Sections for the ${ }^{32} \mathrm{~S}(n, p){ }^{32} \mathrm{P}$ and the ${ }^{34} \mathrm{~S}(n, \alpha){ }^{31} \mathrm{Si}$ Reactions. Phys. Rev. 107, 1363, (1957).

28. Y. Kasugai, et al., Measurement of (n, p) Reaction Cross Sections for Short-lived products $\left(\mathrm{T}_{1 / 2}=0.6\right.$ $\sim 13.8 \mathrm{~s}$ ) by $14 \mathrm{MeV}$ Neutrons. INDC (JPN)-188/U (JAERI-Conf 2001-006), 190, (2001).

29. P.N. Ngoc, et al., Investigations of (n,p), (n,a) and $(n, 2 n)$ reactions around $14 \mathrm{MeV}$, Thesis abstract, prelim. results of all reactions, Institute 3HUNELU Eotvos Lorand Univ., Budapest, Hungary, (1980).

30. W. Schantl, Inst. fuer Isotopenforschung und Kernphysik, Vienna, Austria, (1970) (unpublished).

31. M. Bormann, et al., Measurements of some fast neutron cross sections with the activation method. Conf.: Nuclear Data For Reactors Conf., Paris, 1, 225, (1967).

32. R. Prasad, D.C. Sarkar, C.S. Khurana, Measurement of $(n, p)$ and $(n, \alpha)$ reaction cross sections at $14.8 \mathrm{MeV}$, Nuclear Physics 85, 2, 476-480, (1966).

33. E.B. Paul, R.L. Clarke, Cross section measurements of reactions induced by neutrons of the $14.5 \mathrm{MeV}$ energy, Canadian Journal of Physics31, 267, (1953). 